\title{
Correlación y conversión entre los valores de actividad de la colinesterasa plasmática medida por tres técnicas diferentes
}

\author{
Jaime Carmona-Fonseca ${ }^{1}$
}

Forma de citar Carmona-Fonseca J. Correlación y conversión entre los valores de actividad de la colinesterasa plasmática medida por tres técnicas diferentes. Rev Panam Salud Publica. 2007;22(1):35-40.

RESUMEN Objetivo. Determinar las relaciones matemáticas entre tres técnicas cuantitativas empleadas para medir la actividad de la colinesterasa plasmática, a partir de los valores de referencia ya establecidos para dos poblaciones de Antioquia, Colombia.

Métodos. Diseño descriptivo, transversal y prospectivo. Se conformaron dos muestras independientes representativas de la población laboral adulta (18-65 años), no expuesta a plaguicidas inhibidores de la colinesterasa y vinculada al Seguro Social, del valle de Aburrá (415 personas) y del cercano oriente antioqueño (412 personas), del departamento de Antioquia, Colombia. La colinesterasa plasmática (EC 3.1.1.8) se midió mediante tres técnicas cuantitativas: Michel, $E Q M^{\circledR}$ y Monotest ${ }^{\circledR}$. Se elaboraron ecuaciones de regresión que relacionaran entre sí los resultados de las tres técnicas estudiadas.

Resultados. Se definieron seis ecuaciones de regresión lineal simple que permitieron relacionar los resultados de las tres técnicas de medición de colinesterasa plasmática analizadas. La correlación entre las técnicas fue moderada (r: de 0,686 a 0,771), pero aumentó (r: de 0,744 a 0,811) al eliminar $12(1,5 \%)$ valores extremos. Las asociaciones entre las técnicas fueron altamente significativas $(\mathrm{P}<0,001)$. Las ecuaciones $\mathrm{Y}=\mathrm{a}+\mathrm{bX}$ ajustadas son: $E Q M(U / m L)=0,40773+1,8757$ (Michel [delta $p H /$ hora]); Michel (delta $p H /$ hora $)=0,25799+0,33871(E Q M[U / m L]) ;$ Monotest $(U / L)=462,0+4565,0(\mathrm{Mi}-$ chel [delta $\mathrm{pH} /$ hora]); Michel (delta $\mathrm{pH} /$ hora) $=0,42956+0,00012125$ (Monotest [U/L]); $\operatorname{EQM}(\mathrm{U} / \mathrm{mL})=0,75333+0,00031056$ (Monotest $[U / L]) ; y$ Monotest $(U / L)=262,0+$ $2118,0(E Q M[U / m L])$.

Conclusiones. Los modelos propuestos permiten convertir los valores de actividad de la colinesterasa medida con las tres técnicas estudiadas.

Palabras clave Colinesterasas, butirilcolinesterasa, pruebas de química clínica, Colombia.

Las colinesterasas eritrocitaria (acetilcolinesterasa) (EC 3.1.1.7) y plasmá-

\footnotetext{
Facultad de Medicina, Grupo de Malaria, Universidad de Antioquia, Calle 62 No. 52-59, Laboratorio 610, Medellín, Colombia, Teléfono/fax (574) 210 6487. Correo electrónico: jaimecarmonaf@hotmail. com
}

tica (colinesterasa sérica, seudocolinesterasa o butirilcolinesterasa) (EC 3.1.1.8) se encuentran en el plasma y en casi todos los tejidos del organismo. En algunos — como en el tejido hepáticoestán en mayores concentraciones, mientras que en otros - como en los tejidos que componen el sistema ner- vioso central y periférico-, en mucho menor concentración (1). Casi todos los casos de intoxicaciones agudas causadas por plaguicidas se deben a los insecticidas órganofosforados y carbámicos, que provocan la inhibición precoz e intensa de las colinesterasas eritrocitaria y plasmática. Por ello, el nivel de 
la actividad enzimática es uno de los mejores indicadores biológicos del efecto de la exposición aguda o crónica a los plaguicidas inhibidores de la colinesterasa (PIC). Cuando la exposición a PIC es prolongada y de intensidad leve se recomienda medir la enzima eritrocitaria y cuando es por corto tiempo e intensa, se prefiere medir la enzima plasmática (2).

En Colombia, las intoxicaciones por PIC constituyen un importante problema de salud. Entre 1993 y 1997 se atendieron 70357 personas expuestas a PIC y 6,1\% de ellas presentaron valores bajos de colinesterasa (mínimo: 5,2\% en 1997; máximo: 7,4\% en 1996), mientras que en el período 1998-2001 se encontraron valores alterados de colinesterasa en 7,6\% de 25242 trabajadores expuestos a PIC (3-9).

Después de Brasil y México, Colombia es el país latinoamericano que emplea mayor cantidad de plaguicidas (7) y se espera un incremento en los próximos años, tanto en su empleo como en su producción (8). En América Central, la tasa de incidencia de intoxicaciones por PIC fue de 6,32 por 100000 habitantes en 1992 y aumentó hasta 19,50 por 100000 habitantes en 2000, con una tasa de letalidad de alrededor de 11\% (10). En México, además de la exposición de personas a plaguicidas - especialmente en el sector agrícola (11) - se ha informado también de la contaminación ambiental con estas sustancias (12). En Costa Rica se han detectado vegetales contaminados con estos compuestos (13), mientras en Brasil, la exposición de las personas a los plaguicidas (organofosforados y carbamatos) (14) y la contaminación de alimentos y el medio ambiente han sido objeto de análisis $(14,15)$. En Bolivia se considera que el nivel de intoxicación ocupacional por plaguicidas entre los agricultores es grave (16).

La contaminación por plaguicidas afecta también a los trabajadores de los Estados Unidos de América, tanto en las zonas agrícolas (17) como en los centros urbanos (18). A pesar de la prohibición de utilizar este tipo de compuestos en las zonas residenciales estadounidenses - lo que ha permitido reducir considerablemente la inci- dencia de intoxicaciones asociadas con sustancias organofosforadas desde 1995 (19)_, se mantiene una elevada contaminación ambiental en algunos estados, como Florida (20).

En los últimos años se han establecido valores de referencia de la actividad de esta enzima en poblaciones laborales de Aburrá y Oriente, dos regiones del departamento de Antioquia, Colombia, tanto en la sangre - medida por la técnica semicuantitativa de Lovibond ${ }^{\circledR}(21)$ - como en los eritrocitos - determinada por dos procedimientos cuantitativos: Michel y $\mathrm{EQM}^{\circledR}(22)$ - y en el plasma - calculada por tres técnicas cuantitativas: Michel, EQM y Monotest $^{\circledR}$ (23)—. También se describió el comportamiento de las colinesterasas eritrocitaria y plasmática en mujeres trabajadoras según el estado de sus hormonas sexuales (24) y se determinaron los valores de referencia de la hemoglobina $y$ el hematocrito en una muestra de trabajadores no expuestos a PIC (25). Más recientemente se publicaron los resultados del análisis de regresión entre dos técnicas (Michel y EQM) empleadas para la medición de la concentración de colinesterasa eritrocitaria tración de colinesterasa en la sangre, los eritrocitos y el plasma (27). Los valores promedio de la colinesterasa plasmática en las regiones de Aburrá y Oriente resultaron similares, tanto con el método de Michel como con EQM $(P>0,05)$, pero no con Monotest $(P=$ 0,0117) (cuadro 1).

En la actualidad se emplean numerosas técnicas para medir la concentración de la colinesterasa en los eritrocitos, la sangre y el plasma. Sin embargo, debido al uso no regulado de esas técnicas se hace difícil comparar los resultados (26) y sobre la relación entre la concen-

de diferentes investigaciones. Por ello es conveniente disponer de modelos matemáticos que permitan convertir los valores establecidos mediante diferentes procedimientos, ya sea para fines epidemiológicos o clínicos. Entre los antecedentes más importantes en este sentido, Da Silva y Midio propusieron un método de conversión para tres técnicas de medición de la concentración de colinesterasa: la de Ellman para sangre y plasma, la de Michel para plasma y eritrocitos y la de Edson para sangre (28); Newman y Que Hee establecieron un algoritmo para convertir las concentraciones de colinesterasa plasmática calculadas mediante un analizador producido por la firma Du Pont (Delaware, Estados Unidos), la técnica ChE-tel, de Pfizer (Nueva York, Estados Unidos) y la técnica de Michel (29); Groff, Kaminskis y Ellin lograron relacionar las unidades de actividad enzimática de la colinesterasa medida por la técnica de Michel en sus variantes manual y automática (30); Morse convirtió los valores de colinesterasa determinados mediante las técnicas de Michel y de Garry y Routh (31); y Grainger y colaboradores correlacionaron los valores obtenidos mediante diversas técnicas manuales y automatizadas (32). Estos informes demuestran que es posible y conveniente disponer de modelos matemáticos que permitan hacer estas conversiones, siempre que se mantengan las condiciones específicas de laboratorio necesarias.

El objetivo del presente trabajo fue determinar las relaciones matemáticas entre tres técnicas cuantitativas empleadas para medir la actividad de la colinesterasa plasmática, a partir de los valores de referencia ya establecidos para dos poblaciones de Antioquia, Colombia.

CUADRO 1. Valores de referencia (promedio \pm desviación estándar) de la actividad de la colinesterasa plasmática en las regiones estudiadas, según diferentes técnicas de medición

\begin{tabular}{lccc}
\hline \multicolumn{1}{c}{ Técnica (unidades) } & Aburrá & Oriente & $P$ \\
\hline Michel (deltas de pH/hora) & $1,111 \pm 0,264$ & $1,125 \pm 0,243$ & 0,689418 \\
$\mathrm{EQM}^{\circledR}(\mathrm{U} / \mathrm{mL})$ & $2,546 \pm 0,631$ & $2,476 \pm 0,589$ & 0,197889 \\
Monotest $^{\circledR}(\mathrm{U} / \mathrm{L})$ & $5743 \pm 1662$ & $5459 \pm 1585$ & 0,011701 \\
\hline
\end{tabular}

Fuente: Elaborado a partir de la referencia 23. 


\section{MATERIALES Y MÉTODOS}

Se empleó un diseño descriptivo, transversal y prospectivo. Se conformaron dos muestras independientes representativas de la población laboral adulta, de 18 a 65 años de edad, no expuesta a PIC y vinculada a empresas afiliadas al Seguro Social situadas en el Valle de Aburrá (415 personas) y en el cercano oriente antioqueño (412 personas). Los detalles metodológicos del estudio se publicaron anteriormente (21-23).

En Aburrá y Oriente se concentra más de $80 \%$ de la población laboral activa afiliada al Seguro Social en Antioquia. Mediante entrevistas a los responsables de salud ocupacional de cada empresa se comprobó que no se usaban productos con inhibidores de la colinesterasa y se excluyeron los trabajadores que manifestaron utilizar productos con esas sustancias en sus puestos de trabajo, en el hogar o en cualquier otro sitio.

Para calcular el tamaño de la muestra se consideró: a) 0,758 como valor de la media poblacional; b) 0,082 como desviación estándar de la población; c) 0,008 como error de muestreo $(e=$ error aceptado por el investigador multiplicado por la media poblacional, en por ciento); y d) el nivel de significación se fijó en $5 \%(\alpha=0,05)$. Como resultado, el tamaño muestral para Aburrá resultó de 403 y para Oriente, de 402.

\section{Técnicas químicas para medir la colinesterasa plasmática}

A cada trabajador se le realizaron mediciones de colinesterasa plasmática mediante una técnica potenciométrica (Michel) y dos técnicas colorimétricas (EQM y Monotest). Las características básicas de las técnicas empleadas para medir la colinesterasa en plasma son (cuadro 2):

- Técnica de Michel: es un método potenciométrico basado en la medición de la variación del pH. La enzima produce ácido a partir de la acetilcolina, la acetil- $\beta$-metilcolina o

CUADRO 2. Características de las técnicas empleadas para medir la actividad de la colinesterasa plasmática ${ }^{a}$

\begin{tabular}{|c|c|c|c|}
\hline Característica & Michel & $\mathrm{EQM}^{\mathrm{Bb}}$ & Monotest $^{\circledR}$ \\
\hline Tipo de técnica & Potenciométrica & $\begin{array}{l}\text { Espectrofotométrica (lectura } \\
\text { a } 440 \mathrm{~nm} \text { ) }\end{array}$ & $\begin{array}{l}\text { Espectrofotométrica (lectura } \\
\text { a } 405 \mathrm{~nm} \text { ) }\end{array}$ \\
\hline \multirow[t]{2}{*}{$\begin{array}{l}\text { Principio de } \\
\text { análisis }\end{array}$} & \multirow[t]{2}{*}{ Medición del pH } & $\begin{array}{l}\text { Colorimétrico cinético o de } \\
\text { punto final }\end{array}$ & $\begin{array}{l}\text { Cinético, cambio de color } \\
\text { por variación del pH }\end{array}$ \\
\hline & & $\begin{array}{l}\text { a) Propionilcolina }+\mathrm{H}_{2} \mathrm{O} \stackrel{\mathrm{CE}}{\longrightarrow} \\
\text { propionato + tiocolina }\end{array}$ & $\begin{array}{l}\text { a) Butiriltiocolina }+\mathrm{H}_{2} \mathrm{O} \stackrel{\mathrm{CE}}{\longrightarrow} \\
\text { butirato + tiocolina }\end{array}$ \\
\hline Reacción & $\begin{array}{l}\text { Acetilcolina } \underset{\text { colina }+ \text { acetato }}{\stackrel{\mathrm{CE}^{\mathrm{c}}}{\longrightarrow}}+\mathrm{H}^{\prime}\end{array}$ & $\begin{array}{l}\text { b) Tiocolina }+ \text { DNTBd }^{d} \longrightarrow \\
\text { ácido 5-tio-2-nitrobenzoico }+ \\
\text { mezcla de disulfuros }\end{array}$ & $\begin{array}{l}\text { b) Tiocolina + DNTB } \longrightarrow \\
\text { 2-nitro-5-mercaptobenzoato }\end{array}$ \\
\hline Muestra & Plasma & Plasma & Plasma \\
\hline $\begin{array}{l}\text { Tiempo de } \\
\text { reacción }\end{array}$ & 60 minutos & 3 minutos & 30 segundos \\
\hline Volumen & $20 \mu \mathrm{L}$ & $10 \mu \mathrm{L}$ & $20 \mu \mathrm{L}$ \\
\hline Temperatura & $25^{\circ} \mathrm{C}$ & $25^{\circ} \mathrm{C}$ & $25^{\circ} \mathrm{C}$ \\
\hline $\begin{array}{l}\text { pH del tampón } \\
\text { para plasma }\end{array}$ & 8,00 & $\begin{array}{l}\text { Los reactivos autoajustan } \\
\text { el pH }\end{array}$ & Los reactivos ajustan el pH \\
\hline
\end{tabular}

${ }^{a}$ Aparecen las condiciones seleccionadas para nuestras mediciones.

b Según el método de Elman modificado (21-23).

c CE: colinesterasa.

c DTNB: ácido ditiobisnitrobenzoico.

la butirilcolina en una solución tampón estándar durante un tiempo determinado. Se usó un potenciómetro Metrohm 632 (Herisau, Suiza). La unidad de medida es delta $\mathrm{pH} /$ hora.

- Técnica EQM: es un método espectrofotométrico basado en la medición de la variación del $\mathrm{pH}$. La cantidad de ácido producida por la enzima provoca un cambio de color que se mide mediante un colorímetro con fuente diódica emisora de luz. Se utilizó un fotocolorímetro portátil EQM 176 (EQM Research Inc., Cincinnati, Ohio, Estados Unidos) que se basa en el método de Ellman modificado por Magnoti (21-23). Los resultados se midieron en unidades por mililitro $(\mathrm{U} / \mathrm{mL})$.

- Técnica Monotest: es un método cinético colorimétrico de la firma Boehringer Mannheim (Manheim, Alemania), que mide el cambio de color provocado por la variación del pH. Se usó un espectrofotómetro UV/VIS Perkin Elmer Junior 35 (Perkin Elmer, Inc; Waltham, Massachusetts, Estados Unidos) con el juego de reactivos Monotest ${ }^{\circledR}$ (Boeh- ringer Mannheim). Se expresa en unidades por litro $(\mathrm{U} / \mathrm{L})$.

\section{Análisis estadístico}

Se elaboraron ecuaciones de regresión que relacionaran entre sí los resultados de las tres técnicas estudiadas, mediante los programas Epi Info 6.04 y Stat Graphics Plus v. 7.1. Para ello se comprobó que:

a) Las variables cuantitativas de los conjuntos de datos de cada prueba colinesterásica siguieron una distribución normal, según pruebas de estadística descriptiva, el histograma de frecuencias y las pruebas de normalidad (ji al cuadrado y de Kolmogorov-Smirnov).

b) El tamaño de la muestra fue igual o mayor de 30 en tres de los grupos de edad (menores de 30 años, de 30 a 39 y de 40 a 49 años). Sin embargo, como el tamaño de la muestra fue menor de 30 en los grupos de edad de 50 a 59 y de 60 a 75 años, se optó por emplear el estadístico $t$ para contrastar el coeficiente $b$. 
c) Para calcular las dócimas de los coeficientes $r$ y $b$ se empleó el estadístico $t$, porque la muestra era menor de 30 y el valor $r$ poblacional se asumió como cero. Se realizó el contraste de la hipótesis nula para $r=0$ mediante la prueba de la $t$ con $(n-2)$ grados de libertad.

En todos los casos se aplicó un nivel de significación $P<0,05$.

\section{RESULTADOS}

Se definieron seis ecuaciones de regresión lineal simple que permitieron relacionar los resultados de las tres técnicas de medición de la actividad de la colinesterasa plasmática analizadas (cuadro 3). El análisis de regresión lineal simple mostró una adecuada linealidad entre las técnicas de Michel y EQM $(r=0,740)$, entre EQM y Monotest $(r=0,771)$ y entre Michel y Monotest $(r=0,686)$, con $(n-2)$ grados de libertad. Los coeficientes $a$ (intersección) y $b$ (pendiente) de las ecuaciones seleccionadas influyeron en forma significativa $(P<0,001)$.

Otros modelos lineales, como el multiplicativo, el exponencial y el recíproco, se ajustaron a los datos en forma similar, pero se prefirió utilizar el modelo lineal por su mayor simplicidad.

Al eliminar los 12 pares de datos (1,5\% del los 287 pares) que más alejados se encontraban de la zona de confianza se logró mejorar el ajuste de la recta y alcanzar un valor de $r=0,811$ entre EQM y Monotest; $r=0,797$ entre Michel y EQM, y $r=0,744$ entre Michel y Monotest (cuadro 4). En todas las ecuaciones ajustadas, los coeficientes $a$ y $b$ mantuvieron su significación estadística $(P<0,05)$.

No se encontraron diferencias significativas $(P<0,05)$ entre los coeficientes de correlación para cada par de técnicas al ajustar por los grupos de edad (todas las edades y personas menores de 50 años) y región (Aburrá y Oriente).

CUADRO 3. Ecuaciones no ajustadas de regresión lineal simple $(Y=a+b X)^{a}$ para convertir los valores de la actividad de la colinesterasa plasmática medida por tres técnicas diferentes

\begin{tabular}{lccl}
\hline \multicolumn{1}{c}{ Y (unidades) } & $a$ & $b$ & \multicolumn{1}{c}{ X (unidades) } \\
\hline EQM (U/mL) & 0,566535 & 1,71913 & Michel (delta pH/hora) \\
Michel (delta pH/hora) & 0,312712 & 0,318372 & EQM (U/mL) \\
Monotest (U/L) & 841,0 & 4219,0 & Michel (delta pH/hora) \\
Michel (delta pH/hora) & 0,83014 & 0,000111523 & Monotest (U/L) \\
EQM (U/mL) & 0,856191 & 0,00029119 & Monotest (U/L) \\
Monotest (U/L) & 461,0 & 2039,84 & EQM (U/mL) \\
\hline
\end{tabular}

$\bar{a}$ En la ecuación $\mathrm{Y}=a+b \mathrm{X}$, $a$ es la intersección de la recta y $b$ es la pendiente o coeficiente de regresión. Las unidades de medida son específicas para cada técnica.

CUADRO 4. Ecuaciones ajustadas ${ }^{a}$ de regresión lineal simple $(Y=a+b X)^{b}$ para convertir los valores de la actividad de la colinesterasa plasmática medida por tres técnicas diferentes

\begin{tabular}{lccl}
\hline \multicolumn{1}{c}{ Y (unidades) } & $a$ & $b$ & \multicolumn{1}{c}{ X (unidades) } \\
\hline EQM $(\mathrm{U} / \mathrm{mL})$ & 0,40773 & 1,8757 & Michel (delta pH/hora) \\
Michel (delta pH/hora) & 0,25799 & 0,33871 & EQM (U/mL) \\
Monotest (U/L) & 462,0 & 4565,0 & Michel (delta pH/hora) \\
Michel (delta pH/hora) & 0,42956 & 0,00012125 & Monotest $(\mathrm{U} / \mathrm{L})$ \\
$\mathrm{EQM}(\mathrm{U} / \mathrm{mL})$ & 0,75333 & 0,00031056 & Monotest $(\mathrm{U} / \mathrm{L})$ \\
Monotest $(\mathrm{U} / \mathrm{L})$ & 262,0 & 2118,0 & $\mathrm{EQM}(\mathrm{U} / \mathrm{mL})$ \\
\hline
\end{tabular}

a Se eliminaron los 12 pares de datos extremos.

${ }^{b}$ En la ecuación $Y=a+b X$, a es la intersección de la recta y $b$ es la pendiente o coeficiente de regresión. Las unidades de medida son específicas para cada técnica.

\section{DISCUSIÓN}

Un requisito para poder convertir las mediciones de la actividad de la colinesterasa plasmática realizadas por diferentes técnicas es que los métodos comparados se relacionen entre sí mediante un modelo matemático que exprese en forma adecuada una asociación significativa. En este informe se da cuenta del resultado del análisis de tal asociación mediante regresión lineal. En efecto, se comprobó que existe una moderada correlación, aunque altamente significativa, entre los resultados de la medición enzimática realizada por tres pruebas cuantitativas que miden la actividad de la colinesterasa plasmática (Michel, EQM y Monotest). Esta correlación aumentó cuando se eliminaron $12(1,5 \%)$ valores extremos (outliers) de los 827 pares de mediciones. Las variaciones que sufren los valores de la actividad de la colinesterasa plasmática con cada técnica de medición y su comportamiento en función de la región, el sexo y la edad se presentaron en otro artículo (23).

La correlación lineal es moderada ( $r$ : de 0,686 a 0,771 para datos sin ajustar y $r$ : de 0,744 a 0,811 después de eliminar los valores extremos), lo que puede deberse a que los datos corresponden a mediciones reales de campo y no a mediciones de laboratorio. Se debe destacar que la asociación entre las técnicas fue altamente significativa siempre y que tanto los valores de la intersección (a) como de la pendiente (b) influyeron en el modelo de manera significativa, tanto con los datos brutos como ajustados. El ajuste mediante la eliminación de solamente 1,5\% de los pares de mediciones, por ser valores extremos que se encontraban fuera de la zona de confianza, permitió mejorar notablemente la correlación entre las variables. Un comportamiento similar se encontró al analizar la correlación entre dos pruebas cuantitativas utilizadas para medir la actividad de la colinesterasa eritrocitaria (Michel y EQM), ya que de un valor inicial de $r=0,666$ pasó a $r=0,718$ cuando se excluyeron 12 pares de datos extremos (26).

Es importante subrayar la baja - prácticamente nula - correlación 
encontrada entre las pruebas eritrocitarias y las plasmáticas, tanto en $\mathrm{Abu-}$ rrá como en Oriente y entre hombres y mujeres $(P>0,05)(26)$.

Las condiciones propias en que se hicieron las mediciones de la actividad enzimática en este trabajo (cuadro 2) se deben tener en cuenta para aplicar satisfactoriamente las ecuaciones propuestas, ya que los valores de la actividad enzimática dependen de esas condiciones y los resultados de la conversión pueden variar si las condiciones de laboratorio difieren de las empleadas aquí, que son las más generales y recomendadas.
Las principales conclusiones derivadas de estos resultados son: 1) se desarrolló un modelo matemático que expresa adecuadamente la correlación lineal entre los valores de la actividad de la colinesterasa plasmática medida por tres técnicas diferentes (Michel, EQM y Monotest); 2) esa correlación fue moderada pero fuertemente significativa; y 3) los modelos propuestos permiten convertir los valores de la actividad de la colinesterasa medidas mediante las tres técnicas estudiadas. Estos resultados pueden ser de gran utilidad en Colombia y otros países, ya que las condiciones emplea- das en las mediciones son fácilmente reproducibles.

Se deben realizar estudios en otras regiones colombianas y en otros países de las América, con las mismas técnicas y condiciones empleadas, con el fin de enriquecer la información existente y confirmar o ajustar las ecuaciones propuestas.

Agradecimientos. Al Fondo de Promoción de la Salud Industrial, del Instituto de Seguro Social de Colombia, y a la Universidad de Antioquia, Antioquia, Colombia, por el apoyo financiero brindado a esta investigación.

\section{REFERENCIAS}

1. Guyton AK. Tratado de fisiología médica. $10 .^{a}$ ed. México, D.F.: Interamericana-McGraw Hill; 2000.

2. Henao S, Corey G. Plaguicidas inhibidores de las colinesterasas. Metepec, México: Environmental Careers Organization, Organización Panamericana de la Salud, Organización Mundial de la Salud; 1991. (Serie Vigilancia No. 11).

3. Morales L, Silva E, Ortiz JE, Ramírez P, García A. Intoxicación por plaguicidas en el departamento del Valle del Cauca, 1997. Informe Quincenal Epidemiológico Nacional. 1998;3: 222-5.

4. Idrobo AJ. Intoxicaciones masivas con plaguicidas en Colombia. Biomédica. 1999;19:67-76.

5. Silva E, Morales L, Ortiz JE, Díaz ML. Evaluación epidemiológica de plaguicidas en el departamento del Meta. Programa VEO, 1996 a 1997. Informe Quincenal Epidemiológico Nacional. 2002;7:389-401.

6. Sánchez JF, Morales L, Varona M, Camacho M, Ortiz J, De La Hoz F. Exposición a plaguicidas inhibidores de colinesterasa en 17 departamentos. Informe Quincenal Epidemiológico Nacional. 1997;2:10-2.

7. Varona M, Morales L, Ortiz J, Sánchez J, Cárdenas O, De la Hoz F. Panorama epidemiológico de exposición a plaguicidas inhibidores de la colinesterasa en 17 departamentos del país. Biomédica. 1998;18:22-9.

8. Silva E, Morales L, Ortiz JE. Evaluación epidemiológica de plaguicidas inhibidores de acetilcolinesterasas en Colombia, 1996-1997. Biomédica. 2000;20:200-9.

9. Cárdenas O, Silva E, Morales L, Ortiz J. Estudio epidemiológico de la exposición a plaguicidas organofosforados y carbamatos en siete departamentos colombianos, 1998-2001. Biomédica. 2005;25:170-80.

10. Henao S. Situación epidemiológica de las intoxicaciones agudas con plaguicidas en el istmo centroamericano 1992-2000. Biomédica. 2002; 22(supl 1):39-40.

11. Rendon-Von Osten J, Memije MG, Ortiz A, Soares AM, Guilhermino L. An integrated approach to assess water quality and environmental contamination in the fluvial-lagoon system of the Palizada River, Mexico. Environ Toxicol Chem. 2006;25:3024-34.

12. Rendon von Osten J, Epomex C, TinocoOjanguren R, Soares AM, Guilhermino L. Effect of pesticide exposure on acetylcholinesterase activity in subsistence farmers from Campeche, Mexico. Arch Environ Health. 2004;59:418-25.

13. Nevermann KS, Guzmán EQ. Detección de plaguicidas en vegetales de Costa Rica mediante la inhibición de colinesterasas humanas. Arch Latinoam Nutr. 2004;54:444-8.

14. Caldas ED, Boon PE, Tressou J. Probabilistic assessment of the cumulative acute exposure to organophosphorus and carbamate insecticides in the Brazilian diet. Toxicology. 2006; 222:132-42.

15. Veiga MM, Silva DM, Veiga LB, Faria MV. Análise da contaminação dos sistemas hídricos por agrotóxicos numa pequena comunidade rural do Sudeste do Brasil. Cad Saude Publica. 2006;22:2391-9.

16. Jors E, Morant RC, Aguilar GC, Huici O, Lander F, Baelum J, et al. Occupational pesticide intoxications among farmers in Bolivia: a cross-sectional study. Environ Health (revista en línea). 2006;5:10.

17. Lessenger JE. Fifteen years of experience in cholinesterase monitoring of insecticide applicators. J Agromedicine. 2005;10:49-56.

18. Weyrauch KF, Boiko PE, Keifer M. Building informed consent for cholinesterase monitoring among pesticide handlers in Washington State. Am J Ind Med. 2005;48:175-81.

19. Sudakin DL, Power LE. Organophosphate exposures in the United States: a longitudinal analysis of incidents reported to poison centers. J Toxicol Environ Health. 2007;70:141-7.

20. Wilsont PC, Foos JF. Survey of carbamate and organophosphorous pesticide export from a South Florida (USA) agricultural watershed: implications of sampling frequency on ecological risk estimation. Environ Toxicol Chem. 2006;25:2847-52.

21. Carmona-Fonseca J, Henao S, Garcés R. Valores de referencia de actividad colinesterásica sanguínea en población laboral activa no expuesta a plaguicidas inhibidores de colinesterasa. Rev Fac Nal Salud Publ (Medellín). 2000; 18:55-72

22. Carmona-Fonseca J. Valores de referencia de la actividad de la colinesterasa eritrocitaria según las técnicas de Michel y EQM ${ }^{\circledR}$ en población laboral de Antioquia, Colombia. Rev Panam Salud Pública. 2003;14:316-21.

23. Carmona-Fonseca J. Valores de referencia de colinesterasa plasmática con los métodos de Michel, EQM ${ }^{\circledR}$ y Monotest ${ }^{\circledR}$ en población laboral activa del departamento de Antioquia, Colombia. Biomédica. 2003;23:437-55.

24. Carmona-Fonseca J. Valores de colinesterasas en trabajadoras activas embarazadas, menstruantes, usuarias de anticonceptivos o menopáusicas. Rev Col Obstet Ginec. 2003;54: 146-56.

25. Carmona-Fonseca J. Valores de referencia de hemoglobina y hematocrito en una población laboral colombiana. Acta Med Colomb. 2003; 28:63-70.

26. Carmona-Fonseca J. Correlación y conversión entre valores de colinesterasa eritrocitaria medida con las técnicas de Michel y EQM. Biomédica. 2006;26:546-55.

27. Carmona-Fonseca J. Colinesterasas en sangre total medidas con técnica semicuantitativa y en eritrocitos o plasma, medidas con técnicas cuantitativas: relaciones. Biomédica. 2007;27: 244-56. 
28. Da Silva ES, Midio AF. Interconversion of units of three methods for blood cholinesterases. Med Lav. 1998;89:265-72.

29. Newman MA, Que Hee SS. Interconversion and comparison of the results of three methods for cholinesterase in serum. Clin Chem. 1984;30:308-10.

30. Groff WA, Kaminskis A, Ellin RI. Interconversion of cholinesterase enzyme activity units by the manual delta $\mathrm{pH}$ method and a recommended automated method. Clin Toxicol. 1976;9:353-8.

31. Morse CS. Interconversion of cholinesterase measurements by the delta $\mathrm{pH}$ method of Michel and the DTNB method of Garry and Routh. Clin Toxicol. 1974;7:389-93.

32. Grainger MM, Groff WA, Ellin RI, Arsenal E. Blood cholinesterase values. Correlation ob- tained by automated and manual techniques. Arch Environ Health. 1968;16:821-2.

Manuscrito recibido el 4 de junio de 2006. Aceptado para publicación, tras revisión, el 12 de julio de 2007.

ABSTRACT Objectives. To determine mathematical correlations of three quantitative techniques to measure plasma cholinesterase, using reference values already established for two populations in the department of Antioquia, Colombia.

\section{Correlation and conversion of plasma cholinesterase activity values using three techniques}

Methods. In this descriptive, cross-sectional, prospective study, two independent, representative samples of working adults (18 to 65 years old) were examined. In both samples the adults worked for businesses associated with Colombia's Social Security system. Adults in the two samples had not been exposed to cholinesterase-inhibiting pesticides. The samples were from two neighboring regions of the department of Antioquia: one sample (415 adults) was from the Aburrá Valley, and the other (412 adults) was from Oriente Antioqueño (Eastern Antioquia). Plasma cholinesterase (EC 3.1.1.8) was measured using three quantitative methods: Michel, EQM, and Monotest. Linear regression equations were developed to correlate results of these three techniques.

Results. Six simple linear regression equations were defined to show the relationship of three measurement techniques for plasma cholinesterase. There was a moderate correlation of the three techniques ( $r=0.686$ to 0.771 ), but it increased ( $r=0.744$ to 0.811 ) when $12(1.5 \%)$ outliers were eliminated. Associations among the three techniques were highly significant $(P<0.001)$. The adjusted equations for $\mathrm{Y}=a+b \mathrm{X}$ are: EQM $(\mathrm{U} / \mathrm{mL})=0.40773+1.8757($ Michel [delta $\mathrm{pH} / \mathrm{h}]) ;$ Michel $($ delta $\mathrm{pH} / \mathrm{h})=0.25799+$ $0.33871(\mathrm{EQM}[\mathrm{U} / \mathrm{mL}]) ;$ Monotest $(\mathrm{U} / \mathrm{L})=462.0+4565.0$ (Michel [delta $\mathrm{pH} / \mathrm{h}])$; Michel $($ delta $\mathrm{pH} / \mathrm{h})=0.42956+0.00012125$ (Monotest $[\mathrm{U} / \mathrm{L}]) ; \mathrm{EQM}(\mathrm{U} / \mathrm{mL})=0.75333$ + 0.00031056 (Monotest [U/L]); and Monotest $(\mathrm{U} / \mathrm{L})=262.0+2118.0(\mathrm{EQM}[\mathrm{U} / \mathrm{mL}])$. Conclusions. The proposed mathematical models allow conversion of cholinesterase activity values using the Michel, EQM, and Monotest techniques. These models can be of assistance in Colombia and other countries where a variety of measurement techniques are used, and where it becomes difficult to compare the results of different studies. Having mathematical models available for conversion of established values can be beneficial whether for clinical or epidemiological purposes.

Key words Blood chemical analysis, butyrylcholinesterase, cholinesterase inhibitors, humans, laboratory techniques and procedures, pesticides, Colombia. 